\title{
Complement Topologies
}

\author{
${ }^{1}$ Chika Moore, ${ }^{2}$ Alexander Ilo \\ ${ }^{I}$ Department Of Mathematics, Nnamdi Azikiwe University, P.M.B. Awka. Alexander Ilo \\ ${ }^{2}$ Department Of Mathematics, Paul University, P.M.B. , Awka Anambra State.
}

\begin{abstract}
Let $(X, \tau)$ be a topological space. We consider the collection $\tau^{c}=$ $\left\{G^{c}: G \in \tau\right\}$ of all the $\tau$-closed subsets of $X$. If $\tau^{c}$ is a topology on $X$ then we call $\tau$ a complement topology on $X$. Necessary and sufficient conditions for a topology $\tau$ on $X$ to be a complement topology on $X$ are examined. We proved, among other things, that

1. Any finite topology (topology with a finite cardinality) is a complement topology;

2. A topology $\tau$ on $X$ is a complement topology on $X$ if, and only if, it is closed under arbitrary intersections;

3. The family of the complements of the topologies in a chain of complement topologies on any set $X$ is itself also a chain of complement topologies on $X$.
\end{abstract}

Keywords: Complement Topology, Arbitrary Intersections, Chain of Topologies.

\section{Introduction}

It is known that a topology $\tau$ on a set $X$ is the collection of all the open subsets of $X$. Hence, a topology $\tau$ on a set $X$ is a collection of subsets of $X$ which satisfy the axiom of openness; the standard four conditions. Openness of a subset is therefore relative to the topology under consideration. Some sets which are considered closed in one topology are open in another topology and vice-versa. A question of interest is Can all those sets considered closed with respect to a topology on a set $X$ be precisely the ones considered open with respect to another topology on $X$ ? Of course, we are excluding the trivial cases of the discrete and indiscrete topologies on $X$. This seemingly academic but rather interesting question is the main motivation for this paper.

\section{Main Results|De_Nitions, Properties And Im- Plications}

Definition 2.1 Let $(X, \tau)$ be a topological space and let $\tau^{c}$ be the collection

$$
\tau^{c}=\left\{G^{c}: G \in \tau\right\}
$$

of complements of $\tau$-open sets. Then we call $\tau^{c}$ the complement of the topology $\tau$ on $X$.

Definition 2.2 If $(X, \tau)$ is a topological space and the complement $\tau^{c}$ of $\tau$ is itself also a topology on $X$, we call $\tau$ a complement topology, on $X$.

\section{REMARK}

Since $\tau=\left(\tau^{c}\right)^{c}$, it follows that $\tau$ is a complement topology on $X$ if and only if $\tau^{c}$ is also a complement topology on $X$. It turns out that large classes of topologies are complement topologies.

Theorem 2.1 Every topology on a finite set is a complement topology. 
Proof:

Let $\tau$ be a topology on a finite set $X$ and let $\tau^{c}$ be its complement. Then

1. Clearly both $\emptyset$ and $X$ belong to $\tau^{c}$.

2. Let $\left\{G_{i}\right\}_{i=1}^{n} \subset \tau^{c}$. Then $\bigcap_{i=1}^{n} G_{i}=\left(\bigcup_{i=1}^{n} G_{i}^{c}\right)^{c}$. But $G_{i} \in \tau^{c} \Rightarrow G_{i}^{c} \in \tau$. $\Rightarrow \bigcup_{i=1}^{n} G_{i}^{c} \in \tau . \Rightarrow \bigcap_{i=1}^{n} G_{i}=\left(\bigcup_{i=1}^{n} G_{i}^{c}\right)^{c} \in \tau^{c} . \Rightarrow \tau^{c}$ is closed under finite intersections.

3. Let $\left\{G_{\alpha}\right\}_{\alpha \in \Delta} \subset \tau^{c}$. Then $\bigcup_{\alpha \in \Delta} G_{\alpha}=\left(\bigcap_{\alpha \in \Delta} G_{\alpha}^{c}\right)^{c}$. Now, $G_{\alpha} \in \tau^{c} \Rightarrow$ $G_{\alpha}^{c} \in \tau . \Rightarrow \bigcap_{\alpha \in \Delta} G_{\alpha}^{c} \in \tau$, as finite intersections of sets of $\tau$ belong to $\tau$. (We observe that the intersection cannot be infinite since $X$ is finite.) Hence, since the complement of every set in $\tau$ is collected in $\tau^{c}$, it follows that $\bigcup_{\alpha \in \Delta} G_{\alpha}=\left(\bigcap_{\alpha \in \Delta} G_{\alpha}^{c}\right)^{c} \in \tau^{c}$. This implies that $\tau^{c}$ is also closed under arbitrary unions. Hence the complement of every topology on a finite set is a topology on the set.

\section{Example 2.1}

Let $X=\left\{x_{1}, x_{2}, x_{3}, \cdots, x_{n}\right\}$ be any non-empty finite set and let

$$
\tau=\left\{\emptyset, X,\left\{x_{1}\right\},\left\{x_{1}, x_{2}\right\}\right\}
$$

be a topology on $X$. Then $\tau^{c}=\left\{X, \emptyset,\left\{x_{2}, x_{3}, \cdots, x_{n}\right\},\left\{x_{3}, x_{4}, \cdots, x_{n}\right\}\right\}$ is clearly a topology on $X$.

\section{Example 2.2}

Let $X=\left\{x_{1}, x_{2}, x_{3}, \cdots, x_{n}\right\}$ be a non-empty finite set and let

$$
\tau_{k}=\left\{\emptyset, X,\left\{x_{1}\right\},\left\{x_{1}, x_{2}\right\}, \cdots,\left\{x_{1}, x_{2}, \cdots, x_{k}\right\}\right\} ; 1 \leq k<n .
$$

Then $\tau_{k}$ is a topology on $X$, for all $k$. Now

$$
\tau_{k}^{c}=\left\{X, \emptyset,\left\{x_{2}, \cdots, x_{n}\right\},\left\{x_{3}, \cdots, x_{n}\right\}, \cdots,\left\{x_{k+1}, \cdots, x_{n}\right\}\right\}
$$

is also a topology on $X, 1 \leq k<n$. (This example illustrates the remark after Definition 2.2 above. More of such examples appear at the end of section 3.) The proof of theorem 2.1 above points the way for a more general result.

Theorem 2.2 Let $X$ be any nonempty set and let $\tau$ be a finite topology (topology with finite cardinality) on $X$. Then $\tau$ is a complement topology on $X$.

Corollary 2.1 Let $X$ be an infinite set and let $\tau$ be a topology on $X$. Then the complement $\tau^{c}$ of the topology $\tau$ is itself a topology on $X$ if $\tau$ contains only a finite number of open sets.

\section{Example 2.3}

Let $a, b \in R$ be any two real numbers. Then $\tau=\{\emptyset, R,\{a\},\{b\},\{a, b\}\}$ is a topology on $R$. Without loss of generality, we can let $a<b$. Then the complement

$$
\begin{aligned}
& \tau^{c}=\{\emptyset, R, R-\{a\}, R-\{b\}, R-\{a, b\}\} \\
& =\{\emptyset, R,(-\infty, a) \bigcup(a,+\infty),(-\infty, b) \bigcup(b,+\infty),(-\infty, a) \bigcup(a, b) \bigcup(b,+\infty)\}
\end{aligned}
$$

of $\tau$ is easily seen to be a topology on $R$.

Let $G_{0}=N=\{0,1,2, \cdots\}, G_{1}=\{1,2,3, \cdots\}, G_{2}=\{2,3,4, \cdots\}$. Then $\tau=\left\{\emptyset, G_{k}\right\}_{k=0}^{2}$ is easily seen to be a topology on $N$. The complement of $\tau$, $\tau^{c}=\{\emptyset, N,\{0\},\{0,1\}\}$ is also a topology on $N$. In general if $G_{0}=N, G_{1}=$ $N-\{0\}, G_{2}=N-\{0,1\}, G_{3}=N-\{0,1,2\}, \cdots, G_{n}=N-\{0,1,2, \cdots, n-1\}$, then $\tau=\left\{\emptyset, G_{k}\right\}_{k=0}^{n}$ is a topology on $N$, and its complement $\tau^{c}$ is also a topology on $N$. 
Now, every topology $\tau$ on a finite set is necessarily finite. Hence theorem 2.2 asserts, relative to theorem 2.1, that every finite topology on an infinite set is a complement topology. This raises the following interesting question: Are the finite topologies the only topologies on infinite sets that are complement topology? That is, is a complement topology on an infinite set necessarily finite? The next theorem which answers the above question in the negative provides a characterization of complement topologies.

Theorem 2.3 A topology $\tau$ on a set $X$ is a complement topology if, and only if $\tau$ is closed under arbitrary intersections.

\section{Proof:}

$\Longrightarrow$ Clearly if $\tau$ is a complement topology then it is closed under arbitrary intersections.

$\Longleftarrow$. Let $\tau$ be closed under arbitrary intersections and let $\tau^{c}$ be the complement of $\tau$. We show that $\tau^{c}$ is a topology on $X$. We need only show that $\tau^{c}$ is closed under arbitrary unions, as the other properties of a topology are easily seen to be satisfied by $\tau^{c}$. So, let $\left\{A_{\alpha}: \alpha \in \Delta\right\} \subset \tau^{c}$ be any family of sets of $\tau^{c}$. We consider

$$
\left(\bigcup_{\alpha \in \Delta} A_{\alpha}\right)^{c}=\bigcap_{\alpha \in \Delta} A_{\alpha}^{c}
$$

Clearly $A_{\alpha}^{c} \in \tau$, for all $A_{\alpha} \in \tau^{c}$. Since $\tau$ is, by hypothesis, closed under arbitrary intersections $\bigcap_{\alpha \in \Delta} A_{\alpha}^{c} \in \tau$. Hence the left side of (1) is an element of $\tau$; implying that $\left[\left(\bigcup_{\alpha \in \Delta} A_{\alpha}\right)^{c}\right]^{c}=\left(\bigcup_{\alpha \in \Delta} A_{\alpha}\right) \in \tau^{c}$.

From theorem 2.3, it follows that every discrete topology is a complement topology; and in particular it follows that discrete topologies of in_nite sets (which necessarily contain in_nitely many open sets) are complement topolo- gies. And there are other complement topologies, with in_nitely many open sets, which are not discrete topologies.

Lemma 2.1 (Comparison) Let $\tau_{1}$ and $\tau_{2}$ be any two complement topologies on a set $X$ such that (say) $\tau_{1}$ is weaker than $\tau_{2}$. Then $\tau_{1}^{c}$ is weaker than $\tau_{2}^{c}$.

\section{Application}

Definition 3.1 A family $C=\left\{\tau_{\alpha}\right\}_{\alpha \in \Delta}$ of topologies on a set, $X$, is called a chain of topologies, on $X$, if elements of $C$ are pair-wise comparable, in that for any two topologies, $\tau_{\alpha}$ and $\tau_{\beta}$, in $C$, either $\tau_{\alpha}$ is weaker than $\tau_{\beta}$ or vice versa.

Definition 3.2 Any topology which is an element of a chain $C$ of topologies on a set is called a chain element topology.

Theorem 3.1 Let $X=\left\{x_{1}, x_{2}, x_{3}, \cdots, x_{n}\right\}$ be any non-empty finite set. There exists a finite family of topologies on $X$ forming a chain, such that the family of their complement topologies is also a chain.

Proof:

Let

$$
G_{1}=X-\left\{x_{1}\right\}=\left\{x_{2}, x_{3}, \cdots, x_{n}\right\} .
$$

Then $\tau_{1}=\left\{\emptyset, G_{0}, G_{1}\right\}$ is a topology on $X$.

Let 


$$
\begin{aligned}
& G_{0}=X \\
& G_{1}=X-\left\{x_{1}\right\}=\left\{x_{2}, x_{3}, \cdots, x_{n}\right\} \\
& G_{2}=X-\left\{x_{1}, x_{2}\right\}=\left\{x_{3}, x_{4}, \cdots, x_{n}\right\} .
\end{aligned}
$$

Then $\tau_{2}=\left\{\emptyset, G_{k}\right\}_{k=0}^{2}$ is a topology on $X$, stronger than $\tau_{1}$.

Let

$$
\begin{aligned}
& G_{0}=X \\
& G_{1}=X-\left\{x_{1}\right\}=\left\{x_{2}, x_{3}, \cdots, x_{n}\right\} ; \\
& G_{2}=X-\left\{x_{1}, x_{2}\right\}=\left\{x_{3}, x_{4}, \cdots, x_{n}\right\} ; \\
& G_{3}=X-\left\{x_{1}, x_{2}, x_{3}\right\}=\left\{x_{4}, x_{5}, \cdots, x_{n}\right\} ; \\
& G_{k}=X-\left\{x_{1}, x_{2}, \cdots, x_{k}\right\}=\left\{x_{k+1}, x_{k+2}, \cdots, x_{n}\right\}, \\
& 1 \leq k \leq n \text {. Then } \tau_{k}=\left\{\emptyset, G_{t}\right\}_{t=0}^{k} \text { is a topology on } X \text { finer than } \tau_{k-1} \text {. Hence } \\
& \left\{\tau_{k}\right\}_{k=1}^{n} \text { is a (finite) family of topologies on } X \text { forming a chain in that } \\
& \tau_{1}<\tau_{2}<\cdots<\tau_{n} . \\
& \tau_{1}^{c}=\left\{\emptyset, X,\left\{x_{1}\right\}\right\} ; \\
& \tau_{2}^{c}=\left\{\emptyset, X,\left\{x_{1}\right\},\left\{x_{1}, x_{2}\right\}\right\} \text {, etc. }
\end{aligned}
$$

We also see that

are topologies (in chain) on X.

Proof of Theorem 3.1 can be extended to any set|even if in nite|with a chain of complement topologies. The next corollary states this.

Corollary 3.1 Let $C=\left\{\tau_{\alpha}\right\}_{\alpha \in \Delta}$ be a chain of complement topologies on any set $X$. Then the family $C^{*}=\left\{\tau_{\alpha}^{c}: \tau_{\alpha} \in C\right\}_{\alpha \in \Delta}$ of complements of the topologies in $C$ is also a chain of complement topologies on $X$. Conversely, the family of the complements of the topologies in a chain of complement topologies on any set $X$ is itself also a chain of complement topologies on $X$.

\section{More Examples}

[1] The usual topology $u$ on the set $R$ of real numbers is not closed under arbitrary intersections and is thus not a complement topology.

[2] The usual topology on the Cartesian plane is not closed under arbitrary intersections and is, hence, not a complement topology.

[3] The lower limit (or Sorgenfrey) topology on $R$ is not closed under arbitrary intersections and is also not a complement topology.

[4] Let $X=\left\{x_{1}, x_{2}, \cdots, x_{n}\right\}$ be a finite set, and let $k \in N$ be such that $2 k-1 \leq n$. Then

$$
\tau_{2 k-1}=\left\{\emptyset, X,\left\{x_{1}\right\},\left\{x_{1}, x_{3}\right\}, \cdots,\left\{x_{1}, x_{3}, \cdots, x_{2 k-1}\right\}\right\}
$$

is a topology on $X$, for $1 \leq k \leq\left[\frac{n-1}{2}\right]$. We see also that

$$
\tau_{2 k-1}^{c}=\left\{X, \emptyset,\left\{x_{2}, \cdots, x_{n}\right\},\left\{x_{2}, x_{4}, \cdots, x_{n}\right\}, \cdots,\left\{x_{2}, x_{4}, \cdots, x_{n}\right\}\right\}
$$

is a topology on $X$. 
[5] Let $X=\left\{x_{1}, x_{2}, \cdots, x_{n}\right\}$ be a finite set, and let $k \in N$ be such that $2 k-$ $1 \leq n$. Then $\tau_{2 k-1}=\left\{\emptyset, X, \bigcup_{t=1}^{k}\left\{x_{2 t-1}\right\}\right\}$ is a topology on $X$, for $1 \leq k \leq\left[\frac{n-1}{2}\right]$. Also $\tau_{2 k-1}^{c}=\left\{X, \emptyset, X-\bigcup_{t=1}^{k}\left\{x_{2 t-1}\right\}\right\}$ is a topology on $X ; 1 \leq k \leq\left[\frac{n-1}{2}\right]$.

[6] Let $X=\left\{x_{1}, x_{2}, \cdots, x_{n}\right\}$ be a finite set, $n=m t+r, 0 \leq r<m$. Let $\tau_{m}=$ $\left\{\emptyset, X,\left\{x_{m}\right\},\left\{x_{m}, x_{2 m}\right\}, \cdots,\left\{x_{m}, x_{2 m}, \cdots, x_{t m}\right\}\right\}=\left\{\emptyset, X, \bigcup_{i=1}^{k}\left\{x_{i m}\right\}\right\} ; 1 \leq k \leq$ $t$. Then $\tau_{m}$ is a topology on $X$. And we see that $\left\{X, \emptyset, X-\bigcup_{i=1}^{k}\left\{x_{i m}\right\}\right\} ; 1 \leq$ $k \leq t$ is a topology on $X$.

\section{Remark}

It is known that a topological space $(X, \tau)$ is a $T_{1}$-space if, and only if, singletons are $\tau$-closed subsets of $X$. It is observable from the foregoing that if a topology $\tau$ on a set $X$ is a complement topology then the very sets which are seen as $\tau$-closed are the sets which constitute the open sets of another topology on $X$, with equal cardinality as $\tau$. These imply the following.

Corollary 3.2 If $(X, \tau)$ is a $T_{1}$ topological space, then $\tau$ is a complement topology if, and only if, $\tau$ is the discrete topology of $X$.

\section{Proof:}

Since $(X, \tau)$ is $T_{1}$, singletons of $X$ are $\tau$-closed. Since $\tau$ is a complement topology on $X$ and singletons of $X$ are $\tau$-closed, it follows that singletons are among the $\tau^{c}$-open sets. Hence every subset of $X$ is $\tau^{c}$-open, implying that $\tau^{c}$ is the discrete topology of $X$. Since $\left(\tau^{c}\right)^{c}=\tau$, it follows that $\tau$ is the discrete topology of $X$.

\section{Remark}

That a topology is a complement topology does not imply that it is $T_{1}$. Also, every $T_{1}$-space is not a complement topology. By Corollary 3.2, a $T_{1}$-space which is a complement topology must be a discrete topology. It follows that if a $T_{1}$-space is not discrete then it cannot be a complement topology. For example, the set $R$ of real numbers with its usual topology $u$ is $T_{1}$ but $u$ is not a complement topology. Hence all complement topologies are not $T_{1}$ and all $T_{1}$-spaces are not complement topologies.

\section{References}

[1]. Angus E. Taylor and David C. Lay; Introduction to Functional Anal-ysis; Second Edition, John Wiley and Sons, New York, 1980

[2]. Benjamin T. Sims; Fundamentals of Topology; Macmillan Publish-ing Co., Inc., New York; Collier Macmillan Publishers, London and Canada, 1976.

[3]. Edwards R.E.; Functional Analysis: Theory and Applications; Dover Publications Inc., New York, 1995.

[4]. James R. Munkres; Topology; Second Edition, Prentice-Hall of India Private Limited, New Delhi, 2007.

[5]. Seymour Lipschutz; Theory and Problems of General Topology; Schaum's Series, McGraw-Hill Publications, New York, 1965.

[6]. Sheldon W. Davis; Topology; McGraw-Hill Higher Educa- tion/Walter Rudin Series in Advanced Mathematics, Boston, 2005.

[7]. Sidney A. Morris; Topology Without Tears; July 24, 2016 Version, From Internet. Link: sidney.morris@ gmail.com; and www.sidneymorris.net

[8]. Royden H.L. and Fitzpatrick P.M.; Real Analysis; PHI Learning Private Limited, 4th Edition, 2012

[9]. V.S. Medvedev, E.V. Zhuzhoma Morse-Smale Systems with Few Non-wandering Points; Topology and Its Applications (498-507), Elsevier B.V., 2013

[10]. Simmons, G.F. Introduction to Topology and Modern Analysis; McGraw-Hill, New York, 1963.

[11]. Titchmarsh, E.C. Theory of Functions Second Edition, Oxford Uni- versity Press, Oxford, 1939.

[12]. Sheldon W. Davis Topology; McGraw Hill Higher Education, Boston, 2005.

Chika Moore. "Complement Topologies." IOSR Journal of Mathematics (IOSR-JM) 13.3 (2017): 73-77. 